\title{
Nonseparating spheres and twisted Heegaard Floer homology
}

\author{
YI NI
}

If a 3-manifold $Y$ contains a nonseparating sphere, then some twisted Heegaard Floer homology of $Y$ is zero. This simple fact allows us to prove several results about Dehn surgery on knots in such manifolds. Similar results have been proved for knots in $L$-spaces.

57M27; 57R58

\section{Introduction}

Heegaard Floer homology was introduced by Ozsváth and Szabó [16]. For nullhomologous knots, there is a filtered version of Heegaard Floer homology, called knot Floer homology; see Ozsváth and Szabó [14] and Rasmussen [18]. Basically, if one knows the information about the knot Floer homology of a knot, then one can compute the Heegaard Floer homology of any manifold obtained by Dehn surgery on the knot. However, in general the algebra involved here is too complicated. In order to get useful information, people often assume the ambient manifold has "simple" Heegaard Floer homology, namely, the ambient manifold is an $L$-space.

This paper is motivated by the observation that if the ambient manifold contains a nonseparating sphere, and if we use twisted coefficients over a Novikov ring, then the Heegaard Floer homology of the ambient manifold is even simpler: In this case the twisted Heegaard Floer homology is zero. This observation allows us to prove several results about nullhomologous knots in such ambient manifolds.

In order to state the first theorem, we introduce the concept of "Property G".

Suppose $K$ is a nullhomologous knot in a closed 3-manifold $Y$. Then there is a canonical "zero" slope on $K$. Let $Y_{0}(K)$ be the manifold obtained from $Y$ by the zero surgery on $K$. (In general, let $Y_{r}(K)$ be the manifold obtained from $Y$ by $r$-surgery on $K$.) Gabai proved the following result in [5].

Theorem (Gabai) Let $K$ be a knot in $S^{3}, F$ be a minimal genus Seifert surface for $K$. Let $\hat{F} \subset S_{0}^{3}(K)$ be the surface obtained by capping off $\partial F$ with a disk. Then $\widehat{F}$ is Thurston norm minimizing in $S_{0}^{3}(K)$. Moreover, if $S_{0}^{3}(K)$ fibers over the circle, then $K$ is a fibered knot. 
Our notion of "Property G" is motivated by the above theorem.

Definition 1.1 Suppose $K$ is a nullhomologous knot in a closed 3-manifold $Y$. An oriented surface $F \subset Y$ is a Seifert-like surface for $K$, if $\partial F=K$. When $F$ is connected, we say that $F$ is a Seifert surface for $K$. We also view a Seifert-like surface as a proper surface in $Y-\stackrel{\circ}{v}(K)$.

Definition 1.2 Suppose $M$ is a compact 3-manifold, a properly embedded surface $S \subset M$ is taut if $x(S)=x([S])$ in $H_{2}(M, \partial S), S$ is incompressible and no proper subsurface of $S$ is nullhomologous. Here $x(\cdot)$ is the Thurston norm.

Definition 1.3 Suppose $K$ is a nullhomologous knot in a closed 3-manifold $Y$. We say $K$ has Property $G$, if the following conditions hold:

(G1) any taut Seifert-like surface for $K$ extends to a taut surface in $Y_{0}(K)$ after attaching a disk to its boundary;

(G2) if $Y_{0}(K)$ fibers over $S^{1}$, such that the homology class of the fiber is the extension of the homology class of a Seifert surface $F$ for $K$, then $K$ is a fibered knot, and the homology class of the fiber is $[F]$.

If the first (or second) condition holds, then we say that $K$ has Property G1 (or G2).

It is easy to construct knots that violate Property G. However, if we make some assumption on $Y$ or $K$, then we can get Property G. For example, one can show that nonprime knots have Property G. In [5], Gabai proved that if $K$ is a nullhomologous knot in a reducible manifold $Y$, such that $H_{1}(Y)$ is torsion-free and $Y-K$ is irreducible, then $K$ has Property G. This result has overlap with our Theorem 1.4. Moreover, using Heegaard Floer homology, we can show that if $H F_{\text {red }}(Y)=0$ then $K$ has Property G. (For Property (G2), the proof can be found by Ai and the author in $[9 ; 1]$. The proof for Property (G1) is similar.)

The first main theorem in this paper is Property $\mathrm{G}$ for knots in manifolds that contain nonseparating spheres.

Theorem 1.4 Suppose $Y$ is a closed 3-manifold that contains a nonseparating sphere $S, K$ is a nullhomologous knot in $Y$, such that $Y-K$ is irreducible. Then $K$ has Property $G$.

The next result is about cosmetic surgeries on the above knots, which is an analogue of Ozsváth and Szabó [17, Theorem 9.7]. 
Theorem 1.5 Suppose $Y$ is a closed 3-manifold that contains a nonseparating sphere $S, K$ is a nullhomologous knot in $Y$, such that $Y-K$ is irreducible. If two rational numbers $r, s$ satisfy $Y_{r}(K) \cong \pm Y_{s}(K)$, then $r= \pm s$.

The paper is organized as follows. In Section 2 we define a version of twisted Heegaard Floer homology. In Section 3 we collect some properties of twisted Heegaard Floer homology, especially the nontriviality results. Sections 4 and 5 are devoted to the proofs of our main theorems.

Acknowledgements We are very grateful to David Gabai, Cameron Gordon and Matthew Hedden for helpful communications. This work was carried out when the author was at MIT. The author was partially supported by an AIM Five-Year Fellowship and NSF grant number DMS-0805807.

\section{Preliminaries on twisted Heegaard Floer homology}

In this section, we will set up the version of twisted Heegaard Floer homology we need. Our approach is similar to the sketch by the author in [10]. More general constructions can be found in [15] and Jabuka and Mark [7].

\subsection{Twisted chain complexes}

Let $Y$ be a closed, oriented 3-manifold. Then $(\Sigma, \boldsymbol{\alpha}, \boldsymbol{\beta}, z)$ is a Heegaard diagram for $Y$. We always assume the diagram satisfies a certain admissibility condition so that the Heegaard Floer invariants we are considering are well-defined (see [16] for more details).

Let

$$
\Lambda=\left\{\sum_{r \in \mathbb{R}} a_{r} T^{r} \mid a_{r} \in \mathbb{R}, \#\left\{a_{r} \mid a_{r} \neq 0, r \leq c\right\}<\infty \text { for any } c \in \mathbb{R}\right\}
$$

be the universal Novikov ring, which is actually a field.

Let $\omega$ be a 1 -cycle on $\Sigma$, such that it is in general position with the $\alpha$-and $\beta$-curves. Namely, $\omega=\sum k_{i} c_{i}$, where $k_{i} \in \mathbb{R}$, each $c_{i}$ is an immersed closed oriented curve on $\Sigma$, such that $c_{i}$ is transverse to the $\alpha$ - and $\beta$-curves and $c_{i}$ does not contain any intersection point of $\alpha$-and $\beta$-curves. We also regard $\omega$ as a 1 -cycle in $Y$.

Let $\underline{C F^{\infty}}(Y, \omega ; \Lambda)$ be the $\Lambda$-module freely generated by $[\mathbf{x}, i]$, where $\mathbf{x} \in \mathbb{T}_{\alpha} \cap \mathbb{T}_{\beta}$, $i \in \mathbb{Z}$. If $\phi$ is a topological Whitney disk connecting $\mathbf{x}$ to $\mathbf{y}$, let $\partial_{\alpha} \phi=(\partial \phi) \cap \mathbb{T}_{\alpha}$. We can also regard $\partial_{\alpha} \phi$ as a multiarc that lies on $\Sigma$ and connects $\mathbf{x}$ to $\mathbf{y}$. We define

$$
A(\phi)=\left(\partial_{\alpha} \phi\right) \cdot \omega .
$$


Let

$$
\underline{\partial}: \underline{C F^{\infty}}(Y, \omega ; \Lambda) \rightarrow \underline{C F^{\infty}}(Y, \omega ; \Lambda)
$$

be the boundary map defined by

$$
\underline{\partial}[\mathbf{x}, i]=\sum_{\mathbf{y}} \sum_{\substack{\phi \in \pi_{2}(\mathbf{x}, \mathbf{y}) \\ \mu(\phi)=1}} \#(\mathcal{M}(\phi) / \mathbb{R}) T^{A(\phi)}\left[\mathbf{y}, i-n_{z}(\phi)\right] .
$$

Proposition 2.1 If $\omega_{1}, \omega_{2}$ are two 1 -cycles which are homologous in $Y$, then we have the isomorphism of chain complexes

$$
\underline{C F^{\infty}}\left(Y, \omega_{1} ; \Lambda\right) \cong \underline{C F^{\infty}}\left(Y, \omega_{2} ; \Lambda\right) .
$$

In particular, when $\omega$ is nullhomologous in $Y$, the coefficients are "untwisted".

Proof Since $\omega_{1}, \omega_{2}$ are homologous in $Y, \omega_{1}-\omega_{2}$ is homologous to a linear combination of $\alpha$-curves and $\beta$-curves in $\Sigma$. It is easy to check that $\partial_{\alpha} \phi \cdot \gamma=0$ whenever $\phi$ is a Whitney disk and $\gamma$ is a parallel copy of an $\alpha$ - or $\beta$-curve. Hence we may assume that $\omega_{1}-\omega_{2}$ is nullhomologous in $\Sigma$.

Let $D$ be a $2-$ chain in $\Sigma$ such that $\partial D=\omega_{1}-\omega_{2}$. Consider the map

$$
\begin{aligned}
f: \underline{C F^{\infty}}\left(Y, \omega_{1} ; \Lambda\right) & \rightarrow \underline{C F^{\infty}}\left(Y, \omega_{2} ; \Lambda\right), \\
\mathbf{x} & \mapsto T^{D \cdot \mathbf{x}} \mathbf{x},
\end{aligned}
$$

where $D \cdot \mathbf{x}$ is the cap product of $D$ with the $0-$ chain $\sum x_{i}$ if $\mathbf{x}=\left(x_{1}, \ldots, x_{g}\right)$. We can check that $f$ is a chain map which induces an isomorphism.

The standard construction in Heegaard Floer homology [16] allows us to define the chain complexes $\underline{\widehat{C F}}(Y, \omega ; \Lambda)$ and $\underline{C F^{ \pm}}(Y, \omega ; \Lambda)$. The homologies of the chain complexes are called twisted Heegaard Floer homologies. Proposition 2.1 allows us to regard $\omega$ as a homology class in $H_{1}(Y ; \mathbb{R})$.

This version of twisted Heegaard Floer homology is a special case of the general construction in Ozsváth and Szabó [15, Section 8]. In fact, given a 1-cycle $\omega, \Lambda$ can be viewed as a module over the group ring $\mathbb{Z}\left[H^{1}(Y ; \mathbb{Z})\right]$, where the action of $h \in H^{1}(Y ; \mathbb{Z})$ on $T^{r} \in \Lambda$ is given by

$$
h \cdot T^{r}=T^{r+\langle h, \omega\rangle} .
$$

One can check that the twisted Floer homology defined above is exactly the twisted Floer homology over the module $\Lambda$ as defined in [15, Section 8]. 
Proposition 2.2 Let $Y$ be a 3-manifold, $\mathfrak{s}$ be a $\operatorname{Spin}^{c}$ structure, and $\omega$ be a 1-cycle. Then, there are natural isomorphisms

$$
\underline{\widehat{H F}}^{*}(Y, \omega, \mathfrak{s}) \cong \underline{\widehat{H F}}_{*}(-Y, \omega, \mathfrak{s}), \quad \underline{H F_{ \pm}^{*}}(Y, \omega, \mathfrak{s}) \cong \underline{H F_{*}^{\mp}}(-Y, \omega, \mathfrak{s}) .
$$

Proof As in [15, Proposition 2.5], if $(\Sigma, \boldsymbol{\alpha}, \boldsymbol{\beta})$ is a Heegaard diagram for $Y$, then $(-\Sigma, \boldsymbol{\alpha}, \boldsymbol{\beta})$ is a Heegaard diagram for $-Y$. Suppose $\phi \in \pi_{2}(\mathbf{x}, \mathbf{y})$ for $Y$. Then there is a corresponding $\phi^{\prime} \in \pi_{2}(\mathbf{y}, \mathbf{x})$ for $-Y$. Moreover,

$$
\mathcal{M}_{J_{s}}(\phi) \cong \mathcal{M}_{-J_{s}}\left(\phi^{\prime}\right), \quad \partial_{\alpha}(\phi)=-\partial_{\alpha}\left(\phi^{\prime}\right)
$$

We then have

$$
\left(\partial_{\alpha}(\phi) \cdot \omega\right)_{\Sigma}=\left(\partial_{\alpha}\left(\phi^{\prime}\right) \cdot \omega\right)_{-\Sigma} .
$$

Now we can easily get our conclusion.

\subsection{Twisted chain maps}

Let $(\Sigma, \boldsymbol{\alpha}, \boldsymbol{\beta}, \boldsymbol{\gamma}, z)$ be a Heegaard triple-diagram. Let $\omega$ be a 1 -cycle on $\Sigma$ which is in general position with the $\alpha-, \beta$ - and $\gamma$-curves.

The pants construction [16, Subsection 8.1] gives rise to a four-manifold $X_{\alpha, \beta, \gamma}$ with

$$
\partial X_{\alpha, \beta, \gamma}=-Y_{\alpha, \beta}-Y_{\beta, \gamma}+Y_{\alpha, \gamma} .
$$

By this construction $X_{\alpha, \beta, \gamma}$ contains a region $\Sigma \times \triangle$, where $\triangle$ is a two-simplex with edges $e_{\alpha}, e_{\beta}, e_{\gamma}$. Let $\omega \times[0,1]=\omega \times e_{\alpha} \subset X_{\alpha, \beta, \gamma}$ be the linear combination of properly immersed annuli such that

$$
\omega \times\{0\} \subset Y_{\alpha, \beta}, \quad \omega \times\{1\} \subset Y_{\alpha, \gamma} .
$$

Suppose $\mathbf{x} \in \mathbb{T}_{\alpha} \cap \mathbb{T}_{\beta}, \mathbf{y} \in \mathbb{T}_{\beta} \cap \mathbb{T}_{\gamma}, \mathbf{w} \in \mathbb{T}_{\alpha} \cap \mathbb{T}_{\gamma}$ and $\psi$ is a topological Whitney triangle connecting them. Let $\partial_{\alpha} \psi=\partial \psi \cap \mathbb{T}_{\alpha}$ be the arc connecting $\mathbf{x}$ to $\mathbf{w}$. We can regard $\partial_{\alpha} \psi$ as a multiarc on $\Sigma$. Define

$$
A_{3}(\psi)=\left(\partial_{\alpha} \psi\right) \cdot \omega \text {. }
$$

Let the chain map

$$
\underline{f_{\alpha, \beta, \gamma, \omega \times I}^{\infty}}: \underline{C F^{\infty}}\left(Y_{\alpha, \beta}, \omega \times\{0\} ; \Lambda\right) \otimes_{\mathbb{R}} C F^{\infty}\left(Y_{\beta, \gamma} ; \mathbb{R}\right) \rightarrow \underline{C F^{\infty}}\left(Y_{\alpha, \gamma}, \omega \times\{1\} ; \Lambda\right)
$$

be defined by the formula

$$
\underline{f_{\alpha, \beta, \gamma, \omega \times I}^{\infty}}([\mathbf{x}, i] \otimes[\mathbf{y}, j])=\sum_{\mathbf{w}} \sum_{\substack{\psi \in \pi_{2}(\mathbf{x}, \mathbf{y}, \mathbf{w}) \\ \mu(\psi)=0}} \# \mathcal{M}(\psi) T^{A_{3}(\psi)}\left[\mathbf{w}, i+j-n_{z}(\psi)\right] .
$$


The standard constructions $[16 ; 15]$ allow us to define chain maps introduced by cobordisms.

\subsection{Twisted Knot Floer homology}

Suppose $K$ is a rationally nullhomologous oriented knot in $Y, \xi$ is a relative $\operatorname{Spin}^{c}-$ structure in $\operatorname{Spin}^{c}(Y, K)$ and $\omega$ is a $1-$ cycle in $Y-K$. We can define the twisted knot Floer complex $\underline{C} F K^{\infty}(Y, K, \xi, \omega ; \Lambda)$ as in [17, Section 3]; see also [14; 18]. Recall that the chain complex is generated by the $[\mathbf{x}, i, j]$ 's satisfying

$$
\underline{\mathfrak{s}} w, z(\mathbf{x})+(i-j) \cdot \operatorname{PD}[\mu]=\xi .
$$

Since $K$ is oriented, there is a natural way to extend a vector field representing a relative $\operatorname{Spin}^{c}$-structure in $\operatorname{Spin}^{c}(Y, K)$ to a vector field on $Y$. Let

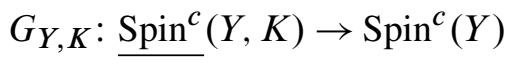

be the induced map of $\operatorname{Spin}^{c}$-structures.

Lemma 2.3 [17, Proposition 3.2] There are natural isomorphisms of chain complexes

$$
\underline{C}_{\xi}\{i=0\} \cong \underline{\widehat{C F}}\left(Y, G_{Y, K}(\xi)\right), \quad \underline{C}_{\xi}\{j=0\} \cong \widehat{\widehat{C F}}\left(Y, G_{Y,-K}(\xi)\right) .
$$

We can construct a Heegaard diagram $(\Sigma, \boldsymbol{\alpha}, \boldsymbol{\beta}, w, z)$ for $(Y, K)$, such that $\beta_{1}=\mu$ is the meridian of $K$, and $\alpha_{1}$ is the only $\alpha$-curve that intersects $\beta_{1}, \alpha_{1} \cap \beta_{1}=\{x\}$. There is a curve $\lambda \subset \Sigma$ which gives rise to the knot $K$. Then $(\Sigma, \boldsymbol{\alpha}, \boldsymbol{\gamma}, z)$ is a diagram for $Y_{m \mu+\lambda}(K)$, where $\gamma_{1}=m \mu+\lambda$ and all other $\gamma_{i}$ 's are small Hamiltonian translations of $\beta_{i}$ 's. Figure 1 (which is a modification of [17, Figure 1]) is the local picture in a cylindrical neighborhood of $\beta_{1}$.

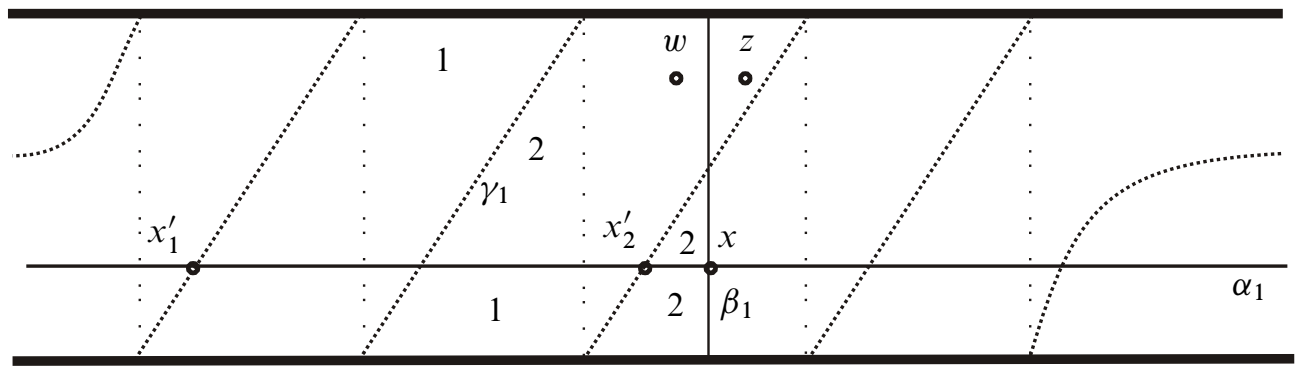

Figure 1: Local picture of the triple Heegaard diagram

As in [17], when $m$ is sufficiently large, one defines a map

$$
\Xi: \operatorname{Spin}^{c}\left(Y_{m \mu+\lambda}(K)\right) \rightarrow \underline{\operatorname{Spin}^{c}}(Y, K)
$$


as follows. If $\mathfrak{t} \in \operatorname{Spin}^{c}\left(Y_{m \mu+\lambda}(K)\right)$ is represented by a point $\mathbf{x}^{\prime}$ supported in the winding region, let $\mathbf{x} \in \mathbb{T}_{\alpha} \cap \mathbb{T}_{\beta}$ be the "nearest point", and let $\psi \in \pi_{2}\left(\mathbf{x}^{\prime}, \Theta\right.$, $\left.\mathbf{x}\right)$ be a small triangle. Then

$$
\Xi(\mathfrak{t})=\underline{\mathfrak{s}}_{w, z}(\mathbf{x})+\left(n_{w}(\psi)-n_{z}(\psi)\right) \cdot \mu .
$$

The reader may also see the author [12] for more discussion on the well-definedness of $\Xi$.

Lemma 2.4 The map $\Xi$ is injective.

Proof Suppose two intersection points $\mathbf{x}_{1}^{\prime}, \mathbf{x}_{2}^{\prime} \in \mathbb{T}_{\alpha} \cap \mathbb{T}_{\gamma}$ are supported in the winding region, and they represent two $\operatorname{Spin}^{c}$-structures $\mathfrak{t}_{1}, \mathfrak{t}_{2} \in \operatorname{Spin}^{c}\left(Y_{m \mu+\lambda}\right)$. Let $\mathbf{x}_{1}, \mathbf{x}_{2} \in \mathbb{T}_{\alpha} \cap \mathbb{T}_{\beta}$ be the nearest points of $\mathbf{x}_{1}^{\prime}, \mathbf{x}_{2}^{\prime}$, and let $\psi_{1}, \psi_{2}$ be the corresponding small triangles.

Assume that $\Xi\left(\mathfrak{t}_{1}\right)=\Xi\left(\mathfrak{t}_{2}\right)$. By Equation (2-2), we have

$$
\underline{\mathfrak{s}}_{w, z}\left(\mathbf{x}_{1}\right)+\left(n_{w}\left(\psi_{1}\right)-n_{z}\left(\psi_{1}\right)\right) \cdot \mu=\underline{\mathfrak{s}}_{w, z}\left(\mathbf{x}_{2}\right)+\left(n_{w}\left(\psi_{2}\right)-n_{z}\left(\psi_{2}\right)\right) \cdot \mu .
$$

Since $\mu$ is nullhomologous in $Y$, then $\mathbf{x}_{1}, \mathbf{x}_{2}$ represent the same $\operatorname{Spin}^{c}$-structure in $\operatorname{Spin}^{c}(Y)$. Hence there is a topological Whitney disk $\phi$ for $\mathbb{T}_{\alpha}, \mathbb{T}_{\beta}$ connecting $\mathbf{x}_{1}$ to $\mathbf{x}_{2}$. Since the $\beta_{1}$-components of $\mathbf{x}_{1}$ and $\mathbf{x}_{2}$ are both $x, \partial \phi$ contains $n_{w}(\phi)-n_{z}(\phi)$ copies of $\beta_{1}$. Let $\psi^{d}=\psi_{1}-\psi_{2}$ (see Figure 1 for an illustration). By (2-3), we have

$$
\underline{\mathfrak{s}}_{w, z}\left(\mathbf{x}_{1}\right)-\underline{\mathfrak{s}}_{w, z}\left(\mathbf{x}_{2}\right)=-\left(n_{w}\left(\psi^{d}\right)-n_{z}\left(\psi^{d}\right)\right) \cdot \mu,
$$

thus

$$
n_{w}(\phi)-n_{z}(\phi)=-\left(n_{w}\left(\psi^{d}\right)-n_{z}\left(\psi^{d}\right)\right) .
$$

So we can glue $\phi$ and $\psi^{d}$ together to get a disk $\varphi^{\prime}$. After a Hamiltonian translation, $\varphi^{\prime}$ becomes a topological Whitney disk $\varphi$ for $\mathbb{T}_{\alpha}, \mathbb{T}_{\gamma}$, connecting $\mathbf{x}_{1}^{\prime}$ to $\mathbf{x}_{2}^{\prime}$. Hence $\mathfrak{t}_{1}=\mathfrak{t}_{2}$.

The following result is the twisted version of [17, Theorem 4.1]. We do not state it in the most generality since the current version suffices for our purpose. Recall that a $U-k n o t$ is a knot in a rational homology three-sphere $Y$ with the property that the induced filtration of $C F^{\infty}(Y, K)$ is trivial.

Proposition 2.5 Let $K \subset Y$ be a rationally nullhomologous knot in a closed, oriented three-manifold, equipped with a frame $\lambda$, and let $\omega$ be a 1 -cycle in $Y-K$. Let $\widehat{A}_{\xi}(Y, K, \omega)=\underline{C}_{\xi}\{\max \{i, j\}=0\}$. Then, for all sufficiently large $m$ and all $\mathfrak{t} \in \operatorname{Spin}^{c}\left(Y_{m \mu+\lambda}(K)\right)$, there is an isomorphism

$$
\Psi_{\mathfrak{t}, m}: \underline{\widehat{C F}}\left(Y_{m \mu+\lambda}(K), \mathfrak{t}, \omega ; \Lambda\right) \rightarrow \widehat{A}_{\Xi(\mathfrak{t})}(Y, K, \omega) .
$$


Proof See [17, Theorem 4.1].

Another result we will need is the following twisted version of [17, Corollary 5.3].

Proposition 2.6 If $K_{2} \subset Y_{2}$ is a $U$-knot, $\omega$ is a 1-cycle in $Y_{1}-K_{1}$, then for each $\xi_{1} \in \operatorname{Spin}^{c}\left(Y_{1}, K_{1}\right)$ and $\mathfrak{s}_{2} \in \operatorname{Spin}^{c}\left(Y_{2}\right)$, there is some $\xi_{2} \in \operatorname{Spin}^{c}\left(Y_{2}, K_{2}\right)$ representing $\overline{\mathfrak{s}_{2}}$, with the property that

$$
\underline{C F K^{\infty}}\left(Y_{1}, K_{1}, \omega, \xi_{1}\right) \cong \underline{C F K^{\infty}}\left(Y_{1} \# Y_{2}, K_{1} \# K_{2}, \omega, \xi_{1} \# \xi_{2}\right)
$$

as $\mathbb{Z} \oplus \mathbb{Z}$-filtered chain complexes.

\section{Properties of twisted Heegaard Floer homology}

In this section, we collect some properties of twisted Heegaard Floer homology. In particular, we prove some nontriviality results following Ozsváth and Szabó [13].

\subsection{Surgery exact sequences}

As in [15], there are surgery exact sequences for twisted Heegaard Floer homology. One of them is as follows (see also Ai and Peters [2]).

Proposition 3.1 Suppose $K \subset Y$ is a knot with frame $\lambda$, and $\omega \subset Y-K$ is a 1-cycle. Then $\omega$ also lies in the manifolds $Y_{\lambda}$ and $Y_{\lambda+\mu}$ obtained by surgeries on $K$. The 2-handle addition cobordism $W$ from $Y$ to $Y_{\lambda}$ naturally contains $\omega \times I$. We can define a chain map induced by $W$,

$$
\underline{f_{W, \omega \times I}^{+}}: \underline{C F^{+}}(Y, \omega ; \Lambda) \rightarrow \underline{C F^{+}}\left(Y_{\lambda}, \omega ; \Lambda\right) .
$$

Similarly, there are two other chain maps induced by the cobordisms $Y_{\lambda} \rightarrow Y_{\lambda+\mu}$ and $Y_{\lambda+\mu} \rightarrow Y$. These maps are the maps in the long exact sequence

$$
\cdots \rightarrow \underline{H F^{+}}(Y, \omega ; \Lambda) \rightarrow \underline{H F^{+}}\left(Y_{\lambda}, \omega ; \Lambda\right) \rightarrow \underline{H F^{+}}\left(Y_{\lambda+\mu}, \omega ; \Lambda\right) \rightarrow \cdots .
$$

Lemma 3.2 Suppose $F$ is a closed surface in a closed manifold $Y$, and $F_{0}$ is a component of $F$ such that its genus greater than or equal to 2. Let $Y^{\prime}$ be the manifold obtained by cutting open $Y$ along $F_{0}$ and regluing by a self-homeomorphism $\varphi$ of $F_{0}$. It is well-known that $\varphi$ can be realized by a product of Dehn twists along a set of curves $\mathcal{C}$ on $F_{0}$. Let $\omega$ be a $1-$ cycle in $Y$ such that $\omega$ is disjoint from $\mathcal{C}$. Then $\omega$ can also be viewed as a 1 -cycle in $Y^{\prime}$. Then we have

$$
\underline{H F^{+}}\left(Y, \omega,[F], \frac{1}{2} x(F) ; \Lambda\right) \cong \underline{H F^{+}}\left(Y^{\prime}, \omega,[F], \frac{1}{2} x(F) ; \Lambda\right) .
$$


Similarly, suppose $F$ is a Seifert-like surface for a knot $K$ in a closed manifold $Y$, and $F_{0}$ is a component of $F$ such that its genus greater than or equal to 2. Let $Y^{\prime}$ be the manifold obtained by cutting $Y$ along $F_{0}$ and regluing by a self-homeomorphism $\varphi$, which can be realized by a product of Dehn twists along a set of curves $\mathcal{C}$ on $F_{0}$. Let $\omega$ be a 1 -cycle in $Y$ such that $\omega$ is disjoint from $\mathcal{C}$. Then $\omega$ can also be viewed as a $1-$ cycle in $Y^{\prime}$. The new knot in $Y^{\prime}$ is still denoted by $K$. Then we have

$$
\widehat{\widehat{H F K}}\left(Y, K, \omega,[F], \frac{1}{2}(x(F)+1) ; \Lambda\right) \cong \underline{\widehat{H F K}}\left(Y^{\prime}, K, \omega,[F], \frac{1}{2}(x(F)+1) ; \Lambda\right) .
$$

Proof The proof is a standard application of the surgery exact sequence and the adjunction inequality; see the second proof of Proposition 3.5 by the author in [8].

\subsection{The presence of a nonseparating sphere}

When there is a nonseparating two-sphere, we have the following properties from [10].

Lemma 3.3 ([10, Lemma 2.1]) Suppose $Y$ contains a nonseparating two-sphere $S$, $\omega \subset Y$ is a closed curve such that $\omega \cdot S \neq 0$. We then have

$$
\widehat{\widehat{H F}}(Y, \omega ; \Lambda)=0, \quad \underline{H F^{+}}(Y, \omega ; \Lambda)=0 .
$$

Lemma 3.4 ([10, Lemma 5.1]) Suppose $Y$ is a closed 3-manifold containing a nonseparating two-sphere $S, K \subset Y$ is a nullhomologous knot, $F$ is a Seifert-like surface for $K$. Let $Y_{0}(K)$ be the manifold obtained by doing 0 -surgery on $K$, and let $\widehat{F}$ be the extension of $F$ in $Y_{0}(K)$. Let $\omega \subset Y-K$ be a $1-$ cycle such that $\omega \cdot S \neq 0$. We then have

$$
\widehat{\widehat{H F K}}\left(Y, K, \omega,[F], \frac{1}{2}(x(F)+1) ; \Lambda\right) \cong \underline{H F^{+}}\left(Y_{0}(K), \omega,[\widehat{F}], \frac{1}{2}(x(F)-1) ; \Lambda\right) .
$$

\subsection{The topmost nontrivial term}

In this subsection we will prove some nontriviality results following the approach in [13]. Although it is possible to prove stronger results, we are satisfied with the current version since it is sufficient for our purpose. We also cite a result about twisted Floer homology and fibered knots.

Lemma 3.5 Suppose $Y$ is a closed 3-manifold with a taut foliation $\mathscr{F}$ which is smooth except possibly along some compact leaves. Then F can be approximated by a positive contact structure $\xi_{+}$and a negative contact structure $\xi_{-}$, and there is a nonempty open subset $U^{*} \subset H^{2}(Y ; \mathbb{R})$ with the following property: For any $h \in U^{*}$, there exists a symplectic form $\Omega$ on $Y \times[-1,1]$, such that $[\Omega]=h \in H^{2}(Y ; \mathbb{R})$, $\left.\Omega\right|_{Y \times\{ \pm 1\}}$ is everywhere positive on $\xi_{ \pm}$. 
Proof By Eliashberg and Thurston [3], we can approximate $\mathscr{F}$ by a positive contact structure $\xi_{+}$and a negative contact structure $\xi_{-}$, and there exists a symplectic form $\Omega$ on $Y \times[-1,1],\left.\Omega\right|_{Y \times\{ \pm 1\}}$ is everywhere positive on $\xi_{ \pm}$. Now if we perturb $\Omega$ by a small closed 2-form on $Y \times[-1,1]$, we still get a symplectic form which is everywhere positive on $\xi_{ \pm}$. This finishes the proof.

Theorem 3.6 Suppose $Y$ is a closed irreducible 3-manifold, $F$ is a taut surface in $Y$. Then there exists a nonempty open set $U \subset H_{1}(Y ; \mathbb{R})$, such that for any $\omega \in U$,

$$
\underline{H F^{+}}\left(Y, \omega,[F], \frac{1}{2} x(F) ; \Lambda\right) \neq 0, \quad \underline{\widehat{H F}}\left(Y, \omega,[F], \frac{1}{2} x(F) ; \Lambda\right) \neq 0 .
$$

Proof By Gabai [4], there exists a taut foliation $\mathscr{F}$ of $Y$, such that $F$ is a union of compact leaves of $\mathscr{F}$, and $\mathscr{F}$ is smooth except possibly along toral components of $F$. By Lemma 3.5 we have a nonempty open subset $U^{*} \subset H^{2}(Y ; \mathbb{R})$ with the property stated there. Let $U \subset H_{1}(Y ; \mathbb{R})$ be the dual of $U^{*}$. Now for any $\omega \in U$, the argument in $\left[13\right.$, Section 4] shows that $\underline{H F^{+}}\left(Y, \omega,[F], \frac{1}{2} x(F) ; \Lambda\right) \neq 0$.

If $\underline{\widehat{H F}}\left(Y, \omega,[F], \frac{1}{2} x(F) ; \Lambda\right)=0$, then the map

$$
U: \underline{H F^{+}}\left(Y, \omega,[F], \frac{1}{2} x(F) ; \Lambda\right) \rightarrow \underline{H F^{+}}\left(Y, \omega,[F], \frac{1}{2} x(F) ; \Lambda\right)
$$

is an isomorphism. Since $\underline{H F^{+}}\left(Y, \omega,[F], \frac{1}{2} x(F) ; \Lambda\right) \neq 0$ and $U^{n} a=0$ for any $a \in \underline{H F^{+}}\left(Y, \omega,[F], \frac{1}{2} x(F) ; \Lambda\right)$ and sufficiently large $n$, we get a contradiction.

The following lemma will be used in the proof of Theorem 3.8.

Lemma 3.7 Suppose $K \subset Y$ is a nullhomologous knot, $Y-K$ is irreducible, $F$ is a taut Seifert-like surface for $K$. Let $J \subset S^{3}$ be a fibered knot with fiber $G$, and let $F^{\prime}$ be the Seifert-like surface for $K \# J$ which is the boundary connected sum of $F$ and $G$. Then if the genus of $J$ is sufficiently large, $Y-\stackrel{\circ}{v}(K \# J)$ admits a longitudinal foliation such that $F^{\prime}$ is a union of compact leaves, and the foliation is smooth except possibly at the toral components of $F^{\prime}$.

Proof The proof is the same as that by the author in [11, Proposition 2.4].

Theorem 3.8 Suppose $K$ is a nullhomologous knot in a closed 3-manifold $Y, Y-K$ is irreducible. Let $F$ be a taut Seifert-like surface for $K$. Then there exists a nonempty open set $U \subset H_{1}(Y ; \mathbb{R})$, such that for any $\omega \in U$,

$$
\widehat{H F K}\left(Y, K, \omega,[F], \frac{1}{2}(x(F)+1) ; \Lambda\right) \neq 0 .
$$


Proof By Lemma 3.7, the complement of $K_{1}=K \# J$ admits a smooth longitudinal foliation with a compact leaf $F_{1}$. So $Y_{0}\left(K_{1}\right)$ admits a taut smooth foliation with a compact leaf $\widehat{F}_{1}$. By Theorem 3.6, there exists a nonempty open set $U_{1} \subset H_{1}\left(Y_{0}\left(K_{1}\right) ; \mathbb{R}\right)$, such that for any $\omega \in U_{1}, \underline{H F^{+}}\left(Y_{0}\left(K_{1}\right), \omega,\left[\widehat{F}_{1}\right], \frac{1}{2}\left(x\left(F_{1}\right)-1\right)\right) \neq 0$.

Let $(M, \gamma)$ be the sutured manifold obtained by cutting $Y$ open along $F_{1}$. Since $J$ is a fibered knot, $(M, \gamma)$ contains a nonseparating product disk $D$. We can cut $Y$ open along $F_{1}$ then reglue by a diffeomorphism $\varphi$ to get a new knot $K^{\prime}$ in a new manifold $Y^{\prime}$, such that $D \cap R_{+}(\gamma)$ and $D \cap R_{-}(\gamma)$ are glued together. Now $D$ becomes a nonseparating annulus $A$ in the complement of $K^{\prime}$, such that $\partial A$ consists of two copies of the meridian of $K^{\prime}$. So $Y^{\prime}$ contains a nonseparating sphere $S$.

The diffeomorphism $\varphi$ can be realized by a product of Dehn twists along a set of curves $\mathcal{C}$ on $F_{1}$. In other words, there exists a link $\mathcal{L} \subset Y-K_{1}$, such that a Dehn surgery on $\mathcal{L}$ yields $Y^{\prime}$. Let

$$
\begin{aligned}
& \rho: H_{1}\left(Y-K_{1}-\mathcal{L} ; \mathbb{R}\right) \rightarrow H_{1}\left(Y-K_{1} ; \mathbb{R}\right)=H_{1}\left(Y_{0}\left(K_{1}\right) ; \mathbb{R}\right), \\
& \rho^{\prime}: H_{1}\left(Y-K_{1}-\mathcal{L} ; \mathbb{R}\right) \rightarrow H_{1}\left(Y^{\prime}-K^{\prime} ; \mathbb{R}\right)=H_{1}\left(Y_{0}^{\prime}\left(K^{\prime}\right) ; \mathbb{R}\right),
\end{aligned}
$$

be the natural inclusion maps. Both $\rho$ and $\rho^{\prime}$ are surjective. Let

$$
\mathcal{V} \subset H_{1}\left(Y^{\prime}-K^{\prime} ; \mathbb{R}\right)
$$

be the codimension 1 subspace defined by

$$
v \cdot[S]=0 .
$$

Let $\omega$ be a $1-$ cycle in $Y-K_{1}-\mathcal{L}$ such that $\omega \in \rho^{-1}(U)-\left(\rho^{\prime}\right)^{-1}(\mathcal{V})$. By Lemma 3.2, we have

$$
\begin{aligned}
& \widehat{\widehat{H F K}}\left(Y, K_{1}, \rho(\omega),\left[F_{1}\right], \frac{1}{2}\left(x\left(F_{1}\right)+1\right)\right) \cong \underline{\widehat{H F K}}\left(Y^{\prime}, K^{\prime}, \rho^{\prime}(\omega),\left[F_{1}\right], \frac{1}{2}\left(x\left(F_{1}\right)+1\right)\right), \\
& \underline{H F^{+}}\left(Y_{0}\left(K_{1}\right), \rho(\omega),\left[\widehat{F}_{1}\right], \frac{1}{2}\left(x\left(F_{1}\right)-1\right)\right) \cong \underline{H F^{+}}\left(Y_{0}^{\prime}\left(K^{\prime}\right), \rho^{\prime}(\omega),\left[\widehat{F}_{1}\right], \frac{1}{2}\left(x\left(F_{1}\right)-1\right)\right) \text {. }
\end{aligned}
$$

By Lemma 3.4,

$\widehat{\widehat{H F K}}\left(Y^{\prime}, K^{\prime}, \rho^{\prime}(\omega),\left[F_{1}\right], \frac{1}{2}\left(x\left(F_{1}\right)+1\right)\right) \cong \underline{H F^{+}}\left(Y_{0}^{\prime}\left(K^{\prime}\right), \rho^{\prime}(\omega),\left[\widehat{F}_{1}\right], \frac{1}{2}\left(x\left(F_{1}\right)-1\right)\right)$. Hence $\underline{\widehat{H F K}}\left(Y, K_{1}, \rho(\omega),\left[F_{1}\right],\left(x\left(F_{1}\right)+1\right) / 2 ; \Lambda\right) \neq 0$.

By the connected sum formula

$$
\widehat{\widehat{H F K}}(Y, K \# J, \rho(\omega) ; \Lambda) \cong \underline{\widehat{H F K}}(Y, K, \rho(\omega) ; \Lambda) \otimes \widehat{H F K}\left(S^{3}, J ; \mathbb{R}\right),
$$

we conclude for any $\omega \in \rho^{-1}(U)-\left(\rho^{\prime}\right)^{-1}(\mathcal{V}), \underline{\widehat{H F K}}(Y, K, \rho(\omega),(x(F)+1) / 2) \neq 0$. Let

$$
i_{*}: H_{1}\left(Y_{0}(K) ; \mathbb{R}\right)=H_{1}(Y-K ; \mathbb{R}) \rightarrow H_{1}(Y ; \mathbb{R})
$$


be the natural map which is a projection. Let

$$
U=i_{*} \rho\left(\rho^{-1}(U)-\left(\rho^{\prime}\right)^{-1}(\mathcal{V})\right) .
$$

Then $U$ is the nonempty open set we need.

The following result is a twisted version of a theorem due to Ghiggini [6] and the author [9].

Theorem 3.9 [10, Theorem 2.2] Suppose $K$ is a nullhomologous knot in a closed, oriented, connected 3-manifold $Y, Y-K$ is irreducible, and $F$ is a genus $g$ Seifert surface for $K$. Let $\omega \subset Y-K$ be a $1-$ cycle. If

$$
\widehat{H F K}(Y, K, \omega,[F], g ; \Lambda) \cong \Lambda,
$$

then $K$ is fibered, and $F$ is a fiber of the fibration.

\section{Property G}

This section is devoted to the proof of Theorem 1.4, which is a direct corollary of the properties listed in the last section.

Proof of Theorem 1.4 We first prove Property (G1). If $F$ is a taut Seifert-like surface for $K$, by Theorem 3.8 we can find a 1 -cycle $\omega \subset Y-K$, such that $\omega \cdot S \neq 0$ and

$$
\widehat{H F K}\left(Y, K, \omega,[F], \frac{1}{2}(x(F)+1) ; \Lambda\right) \neq 0 .
$$

Now Lemma 3.4 implies that

$$
\underline{H F^{+}}\left(Y_{0}(K), \omega,[\widehat{F}], \frac{1}{2}(x(F)-1) ; \Lambda\right) \neq 0,
$$

hence $\widehat{F}$ is taut.

Now we prove Property (G2). Suppose $Y_{0}(K)$ fibers over $S^{1}$ with fiber in the homology class $[\widehat{F}]$, where $F$ is a taut Seifert-like surface for $K$. By Property $(\mathrm{G} 1), \hat{F}$ is taut in $Y_{0}(K)$, hence $\widehat{F}$ is isotopic to a fiber of the fibration. Choose a $1-$ cycle $\omega \subset Y-K$, such that $\omega \cdot S \neq 0, \omega \cdot[\widehat{F}] \neq 0$. Since $Y_{0}(K)$ fibers over $S^{1}$, by [2] we have

$$
\underline{H F^{+}}\left(Y_{0}(K), \omega,[\hat{F}], g(F)-1 ; \Lambda\right) \cong \Lambda .
$$

Lemma 3.4 then implies that

$$
\widehat{\hat{H F K}}(Y, K, \omega,[F], g(F) ; \Lambda) \cong \Lambda .
$$

Using Theorem 3.9, we conclude that $K$ is fibered with fiber $F$. 


\section{Cosmetic surgery}

In this section, we will prove Theorem 1.5. Like [17, Section 9], the proof relies on the rational surgery formula of Floer homology. However, our situation here is much simpler. The result we will use is as follows.

Proposition 5.1 Let $Y$ be a closed 3-manifold that contains a nonseparating sphere $S$. Then $K$ is a nullhomologous knot in $Y$, such that $Y-K$ is irreducible. Let $\omega$ be a 1 -cycle in $Y-K$ satisfying $\omega \cdot S \neq 0$. Then there exists a constant $R=R(Y, K, \omega)$, such that

$$
\operatorname{rank}_{\Lambda} \underline{\widehat{H F}}\left(Y_{p / q}(K), \omega ; \Lambda\right)=q R
$$

for any $p / q \in \mathbb{Q}$. Here $p, q \in \mathbb{Z}, q>0, \operatorname{gcd}(p, q)=1$.

The following lemma is an analogue of [17, Theorem 6.1].

Lemma 5.2 Let $Y$ be a closed 3-manifold that contains a nonseparating sphere $S$. Let $K$ be a rationally nullhomologous knot in $Y, \lambda$ a frame on $K$. Let $\omega$ be a 1-cycle in $Y-K$ satisfying $\omega \cdot S \neq 0$. Let

$$
\widehat{\mathbb{A}}(Y, K, \omega)=\bigoplus_{\xi \in \underline{\operatorname{Spin}^{c}(Y, K)}} \widehat{A}_{\xi}(Y, K, \omega) .
$$

Then there is an isomorphism

$$
\widehat{\widehat{H F}}\left(Y_{\lambda}(K), \omega ; \Lambda\right) \cong H_{*}(\widehat{\mathbb{A}}(Y, K, \omega)) .
$$

Proof We claim that for any two frames $\lambda_{1}, \lambda_{2}$ on $K$,

$$
\widehat{\widehat{H F}}\left(Y_{\lambda_{1}}(K), \omega ; \Lambda\right) \cong \underline{\widehat{H F}}\left(Y_{\lambda_{2}}(K), \omega ; \Lambda\right) .
$$

This claim follows from Proposition 3.1 and the fact that

$$
\widehat{\widehat{H F}}(Y, \omega ; \Lambda)=0 .
$$

By the above claim and Proposition 2.5, when $m$ is sufficiently large we have

$$
\widehat{\widehat{H F}}\left(Y_{\lambda}(K), \omega ; \Lambda\right) \cong \bigoplus \widehat{A}_{\Xi(\mathfrak{t})}(Y, K, \omega) .
$$

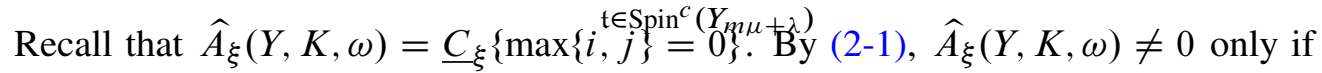
some $\operatorname{Spin}^{c}$-structure $\xi+n \operatorname{PD}[\mu]$ is represented by an intersection point $\mathbf{x} \in \mathbb{T}_{\alpha} \cap \mathbb{T}_{\beta}$. Moreover, by (2-1) and Lemma 2.3, there exists a constant $N_{0}$, such that for any $\mathbf{x}$, if $|n|>N_{0}$, then

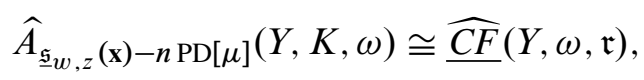


for some $\operatorname{Spin}^{c}$ structure $\mathfrak{r}$ depending on $\mathbf{x}, n$. By Lemma 3.3, the right hand side of the above equation is acyclic.

The analysis in the last paragraph shows that, if $m$ is sufficiently large, then the image of $\Xi$ contains all the $\xi$ such that $\widehat{A}_{\xi}(Y, K, \omega) \neq 0$. Our desired result then follows from (5-1) and Lemma 2.4.

Let $K$ be a nullhomologous knot in $Y$. As in [17, Section 7], $Y_{p / q}(K)$ can be realized by a Morse surgery on the knot $K^{\prime}=K \# O_{q / r} \subset Y^{\prime}=Y \# L(q, r)$, where $O_{q / r}$ is a $U$-knot in $L(q, r)$.

Suppose $\xi \in \operatorname{Spin}^{c}\left(Y^{\prime}, K^{\prime}\right)$, then $\xi$ is the connected sum of two $\operatorname{Spin}^{c}$-structures $\xi_{1} \in$

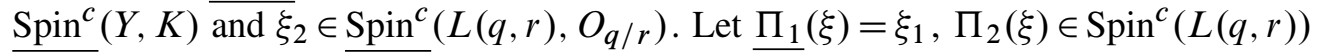
be the $\operatorname{Spin}^{c}$-structure represented by $\xi_{2}$.

Lemma 5.3 There is a bijective map

$$
\underline{\Pi_{1}} \times \Pi_{2}: \underline{\operatorname{Spin}^{c}}\left(Y^{\prime}, K^{\prime}\right) \rightarrow \underline{\operatorname{Spin}^{c}}(Y, K) \times \operatorname{Spin}^{c}(L(q, r)) .
$$

Proof Let

$$
\left(\Sigma_{1}, \boldsymbol{\alpha}_{1}, \boldsymbol{\beta}_{1}, w_{1}, z_{1}\right)
$$

be a doubly pointed Heegaard diagram for $(Y, K)$, and let

$$
\left(\Sigma_{2}, \boldsymbol{\alpha}_{2}, \boldsymbol{\beta}_{2}, w_{2}, z_{2}\right)
$$

be a genus 1 Heegaard diagram for $\left(L(q, r), O_{q / r}\right)$.

We perform the connected sum of $\Sigma_{1}$ and $\Sigma_{2}$ by identifying the neighborhoods of $z_{1}$ and $w_{2}$, hence get a new genus $(g+1)$ surface $\Sigma^{\prime}$. Then

$$
\left(\Sigma^{\prime}, \boldsymbol{\alpha}^{\prime}=\boldsymbol{\alpha}_{1} \cup \boldsymbol{\alpha}_{2}, \boldsymbol{\beta}^{\prime}=\boldsymbol{\beta}_{1} \cup \boldsymbol{\beta}_{2}, w_{1}, z_{2}\right)
$$

is a Heegaard diagram for $\left(Y^{\prime}, K^{\prime}\right)$.

Now $\Pi_{1}$ and $\Pi_{2}$ can be defined as follows. Given $\xi^{\prime} \in \operatorname{Spin}^{c}\left(Y^{\prime}, K^{\prime}\right)$, suppose $\mathbf{x}^{\prime} \in \overline{\mathbb{T}_{\alpha^{\prime}} \cap} \mathbb{T}_{\beta^{\prime}}$ represents the underlying Spin ${ }^{c}$ structure of $\overline{\xi^{\prime} \text {. Then }}$

$$
\xi^{\prime}=\underline{\mathfrak{s}} w_{1}, z_{2}\left(\mathbf{x}^{\prime}\right)+n \cdot \mu^{\prime}
$$

for some $n \in \mathbb{Z}$. Now let $\mathbf{x}_{1}, x_{2}$ be the projections of $\mathbf{x}^{\prime}$ to $\mathbb{T}_{\alpha_{1}} \cap \mathbb{T}_{\beta_{1}}$ and $\mathbb{T}_{\alpha_{2}} \cap \mathbb{T}_{\beta_{2}}$. Then

$$
\begin{gathered}
\underline{\Pi_{1}}\left(\xi^{\prime}\right)=\underline{\mathfrak{s}}_{w_{1}, z_{1}}\left(\mathbf{x}_{1}\right)+n \cdot \mu, \\
\Pi_{2}\left(\xi^{\prime}\right)=\mathfrak{s}_{w_{2}}\left(x_{2}\right) .
\end{gathered}
$$


From the above construction, it is clear that the map $\Pi_{1} \times \Pi_{2}$ is surjective. Now we want to prove the injectivity. In fact, suppose $\xi^{\prime}, \eta^{\prime} \in \underline{\operatorname{Spin}^{c}}\left(Y^{\prime}, K^{\prime}\right)$ satisfy

$$
\underline{\Pi_{1}} \times \Pi_{2}\left(\xi^{\prime}\right)=\underline{\Pi_{1}} \times \Pi_{2}\left(\eta^{\prime}\right) .
$$

Choose a diagram as above, such that there are $\mathbf{x}^{\prime}, \mathbf{y}^{\prime} \in \mathbb{T}_{\alpha^{\prime}} \cap \mathbb{T}_{\beta^{\prime}}$ and $n, m \in \mathbb{Z}$, such that

$$
\underline{\mathfrak{s}} w_{1}, z_{2}\left(\mathbf{x}^{\prime}\right)+n \mu^{\prime}=\xi^{\prime}, \quad \underline{\mathfrak{s}}_{w_{1}, z_{2}}\left(\mathbf{y}^{\prime}\right)+m \mu^{\prime}=\eta^{\prime} .
$$

It follows that $\mathfrak{s}_{w_{1}}\left(\mathbf{x}^{\prime}\right)=\mathfrak{s}_{w_{1}}\left(\mathbf{y}^{\prime}\right)$, so $\mathfrak{s}_{w_{1}}\left(\mathbf{x}_{1}\right)=\mathfrak{s}_{w_{1}}\left(\mathbf{y}_{1}\right)$ and $x_{2}=y_{2}$. Here $\mathbf{x}_{1}, \mathbf{y}_{1}, x_{2}$ and $y_{2}$ are the projections of $\mathbf{x}^{\prime}, \mathbf{y}^{\prime}$ to $\mathbb{T}_{\alpha_{1}} \cap \mathbb{T}_{\beta_{1}}$ and $\mathbb{T}_{\alpha_{2}} \cap \mathbb{T}_{\beta_{2}}$.

Now there is a topological Whitney disk $\phi$ for $\mathbb{T}_{\alpha_{1}}$ and $\mathbb{T}_{\beta_{1}}$ which connects $\mathbf{x}_{1}$ to $\mathbf{y}_{1}$. Since $\underline{\Pi_{1}}\left(\xi^{\prime}\right)=\underline{\Pi_{2}}\left(\eta^{\prime}\right)$, by [14, Lemma 2.5] and (5-2), (5-3) we have

$$
n_{z_{1}}(\phi)-n_{w_{1}}(\phi)=m-n .
$$

Let $\phi^{\prime}$ be a topological Whitney disk whose domain is the connected sum of the domain of $\phi$ and $\Sigma_{2}$. Then $\phi^{\prime}$ connects $\mathbf{x}^{\prime}$ to $\mathbf{y}^{\prime}, n_{z_{2}}\left(\phi^{\prime}\right)=n_{w_{2}}\left(\phi^{\prime}\right)=n_{z_{1}}(\phi)$ and $n_{w_{1}}\left(\phi^{\prime}\right)=n_{w_{1}}(\phi)$. It follows that

$$
n_{z_{2}}\left(\phi^{\prime}\right)-n_{w_{1}}\left(\phi^{\prime}\right)=n_{z_{1}}(\phi)-n_{w_{1}}(\phi)=m-n .
$$

So we have

$$
\underline{\mathfrak{s}}_{w_{1}, z_{2}}\left(\mathbf{x}^{\prime}\right)+n \mu^{\prime}=\underline{\mathfrak{s}}_{w_{1}, z_{2}}\left(\mathbf{y}^{\prime}\right)+m \mu^{\prime},
$$

hence $\xi^{\prime}=\eta^{\prime}$. This proves the injectivity.

Theorem 1.5 does not directly follow from the previous two results. The reason is that $\widehat{H F}(Y, \omega ; \Lambda)$ is not an invariant for $Y$ : it depends on the choice of $\omega$. However, it is not hard to overcome this difficulty.

Proof of Theorem 1.5 Assume there are two rational numbers $p_{1} / q_{1}, p_{2} / q_{2}$ satisfying that there is a homeomorphism

$$
f: Y_{p_{1} / q_{1}} \rightarrow \pm Y_{p_{2} / q_{2}} .
$$

Then $\left|p_{1}\right|=\left|p_{2}\right|$ for homological reason. If $p_{1} / q_{1} \neq p_{2} / q_{2}$, then we can assume

$$
0<q_{1}<q_{2} \text {. }
$$

By Lemma 3.4 and Theorem 3.8, there exists a nonempty set $U \subset H_{1}(Y ; \mathbb{R})$, such that for any $1-$ cycle $\omega \subset Y-K$ representing an element in $U$, one has $\underline{H F^{+}}\left(Y_{0}, \omega ; \Lambda\right) \neq 0$. Thus

$$
\widehat{\widehat{H F}}\left(Y_{0}, \omega ; \Lambda\right) \neq 0,
$$


as argued in the proof of Theorem 3.6.

Since $K$ is nullhomologous, we can identify $H_{1}(Y ; \mathbb{R})$ with $H_{1}\left(Y_{r} ; \mathbb{R}\right)$ for any $r \in \mathbb{Q}-\{0\}$. Let $\mathcal{V}$ be the subspace of $H_{1}(Y ; \mathbb{R})$ defined by the equation $x \cdot S=0$. Choose

$$
\omega \in U \backslash \cup_{n \in \mathbb{Z}} f_{*}^{n}(\mathcal{V}) .
$$

Then $\left(f_{*}^{n} \omega\right) \cdot S \neq 0$ for any $n \in \mathbb{Z}$. By Proposition 5.1,

$$
\operatorname{rank}_{\Lambda} \underline{\widehat{H F}}\left(Y_{p_{1} / q_{1}}, f_{*}^{n} \omega ; \Lambda\right)=q_{1} / q_{2} \operatorname{rank}_{\Lambda} \underline{\widehat{H F}}\left(Y_{p_{2} / q_{2}}, f_{*}^{n} \omega ; \Lambda\right)
$$

for any $n \in \mathbb{Z}$. Moreover, since $f: Y_{p_{1} / q_{1}} \rightarrow \pm Y_{p_{2} / q_{2}}$ is a homeomorphism, using Proposition 2.2 if necessary, we have

$$
\operatorname{rank}_{\Lambda} \underline{\widehat{H F}}\left(Y_{p_{1} / q_{1}}, f_{*}^{n} \omega ; \Lambda\right)=\operatorname{rank}_{\Lambda} \underline{\widehat{H F}}\left(Y_{p_{2} / q_{2}}, f_{*}^{n+1} \omega ; \Lambda\right) .
$$

Thus we get

$$
\operatorname{rank}_{\Lambda} \underline{\widehat{H F}}\left(Y_{p_{1} / q_{1}}, f_{*}^{n} \omega ; \Lambda\right)=\left(\frac{q_{1}}{q_{2}}\right)^{n} \operatorname{rank}_{\Lambda} \underline{\widehat{H F}}\left(Y_{p_{1} / q_{1}}, \omega ; \Lambda\right) .
$$

By Proposition 5.1 and (5-4),

$$
\operatorname{rank}_{\Lambda} \underline{\widehat{H F}}\left(Y_{p_{1} / q_{1}}, \omega ; \Lambda\right)=q_{1} \operatorname{rank}_{\Lambda} \underline{\widehat{H F}}\left(Y_{0}, \omega ; \Lambda\right) \neq 0,
$$

so $0<\operatorname{rank}_{\Lambda} \underline{\widehat{H F}}\left(Y_{p_{1} / q_{1}}, f_{*}^{n} \omega ; \Lambda\right)<1$ when $n$ is sufficiently large, a contradiction.

\section{References}

[1] Y Ai, Y Ni, Two applications of twisted Floer homology, Int. Math. Res. Not. (2009) 3726-3746 MR2539188

[2] Y Ai, T D Peters, The twisted Floer homology of torus bundles, Algebr. Geom. Topol. 10 (2010) 679-695 MR2606797

[3] Y M Eliashberg, W P Thurston, Confoliations, University Lecture Series 13, American Mathematical Society (1998) MR1483314

[4] D Gabai, Foliations and the topology of 3-manifolds, J. Differential Geom. 18 (1983) 445-503 MR723813

[5] D Gabai, Foliations and the topology of 3-manifolds, III, J. Differential Geom. 26 (1987) 479-536 MR910018

[6] P Ghiggini, Knot Floer homology detects genus-one fibred knots, Amer. J. Math. 130 (2008) 1151-1169 MR2450204

[7] S Jabuka, TE Mark, Product formulae for Ozsváth-Szabó 4-manifold invariants, Geom. Topol. 12 (2008) 1557-1651 MR2421135 
[8] Y Ni, Sutured Heegaard diagrams for knots, Algebr. Geom. Topol. 6 (2006) 513-537 MR2220687

[9] Y Ni, Knot Floer homology detects fibred knots, Invent. Math. 170 (2007) 577-608 MR2357503

[10] Y Ni, Heegaard Floer homology and fibred 3-manifolds, Amer. J. Math. 131 (2009) 1047-1063 MR2543922

[11] Y Ni, Link Floer homology detects the Thurston norm, Geom. Topol. 13 (2009) 29913019 MR2546619

[12] Y Ni, Thurston norm and cosmetic surgeries, from: "Low-dimensional and symplectic topology”, (M Usher, editor), Proc. Sympos. Pure Math. 82, Amer. Math. Soc., Providence, RI (2011) 53-63 MR2768653

[13] P Ozsváth, Z Szabó, Holomorphic disks and genus bounds, Geom. Topol. 8 (2004) 311-334 MR2023281

[14] P Ozsváth, Z Szabó, Holomorphic disks and knot invariants, Adv. Math. 186 (2004) 58-116 MR2065507

[15] P Ozsváth, Z Szabó, Holomorphic disks and three-manifold invariants: properties and applications, Ann. of Math. 159 (2004) 1159-1245 MR2113020

[16] P Ozsváth, Z Szabó, Holomorphic disks and topological invariants for closed threemanifolds, Ann. of Math. 159 (2004) 1027-1158 MR2113019

[17] P Ozsváth, Z Szabó, Knot Floer homology and rational surgeries, Algebr. Geom. Topol. 11 (2011) 1-68 MR2764036

[18] J A Rasmussen, Floer homology and knot complements, PhD thesis, Harvard University (2003) MR2704683 arXiv:math.GT/0306378

Department of Mathematics, 253-37, California Institute of Technology 251 Sloan Hall, 1200 E California Blvd, Pasadena, CA 91125, USA

yini@caltech.edu

http://www.its.caltech.edu/ yini/

Received: 1 September 2010 